\title{
USO DA RADIAÇÃO GAMA NA INIBIÇÃO DO ESCURECIMENTO DE MANDIOCA (Manihot utilissima Pohl) IN NATURA, SEM CASCA
}

\author{
PEDRO RAMOS DA COSTA NETO * \\ MARTA HELENA FILLE SPOTO ** \\ RACHEL ELIZABETH DOMARCO **
}

\begin{abstract}
Nesse trabalho tratou-se mandioca recém-colhida com radiação gama nas doses de 2; 4; 6; 8 e 10 kGy para evitar o escurecimento enzimático. As amostras irradiadas foram mantidas durante 9 dias a temperatura ambiente e avaliadas através da determinação de cor e análise sensorial. No segundo dia de armazenamento, o controle já apresentou manchas escuras e alterações nas características organolépticas, enquanto as amostras irradiadas com doses de 2 a 6 kGy apresentaram ótima aparência geral e aceitabilidade. Entretanto, com nove dias de armazenamento, as amostras controle $e$ as irradiadas com 2 a 6 kGy estavam impróprias para consumo, enquanto que as amostras irradiadas com 8 e 10 kGy não apresentavam escurecimento e conservaram suas características sensoriais.
\end{abstract}

\section{INTRODUÇÃO}

A preservação de alimentos por irradiação ionizante tem sido assunto de extensivas pesquisas nos últimos anos, devido aos seus benefícios e eficácia. Estudos têm demonstrado que a aplicação de radiação gama em dose não superior a $10 \mathrm{kGy}$ garante total salubridade ao alimento

* Professor do Centro Federal de Educação Tecnológica do Paraná, Departamento de Química e Biologia, Curitiba, PR.

** Pesquisadoras do Centro de Energia Nuclear na Agricultura da Universidade de São Paulo, Seção de Entomologia, Piracicaba - SP. 
irradiado $(3,6,8,9,11,16)$, além de poder ser usada para diminuir a atividade enzimática e ser aplicada para controle do escurecimento no processamento de alimentos $(9,16)$. Com esse propósito a irradiação comercial de alimentos poderia proporcionar aumento da vida de prateleira de produtos perecíveis, como por exemplo a mandioca. Segundo BALAGOPALAN et al. (1) a mandioca, depois de colhida, apresenta mudanças bioquímicas seguidas de infestações microbiológicas, tornandose imprópria para o consumo humano em poucos dias. Inicialmente aparecem pontos e manchas azul-escuros ou marrons na periferia do córtex, que se propagam para as células parenquimais, ocasionando redução de amido, umidade e aumento do conteúdo de açucar. Nessas reações estão envolvidas enzimas celulase, amilase, pectinase, peroxidase e polifenol-oxidase, sendo o aumento da atividade destas duas últimas, responsável pelo escurecimento. A atividade dessas enzimas pode ser retardada ou inativada através de tratamento térmico ou congelamento (1).

$\mathrm{Na}$ avaliação de produtos irradiados, o emprego de testes sensoriais pode oferecer várias informações de grande valor para a apreciação do alimento irradiado, tais como aparência, sabor, cor etc, que se correlacionam de maneira satisfatória com as alterações de natureza química e bacteriológica (15).

Este trabalho teve como objetivo demonstrar que a irradiação de mandioca com raios gama evita o escurecimento enzimático e conserva suas características sensoriais.

\section{MATERIAL E MÉTODOS}

\subsection{OBTENÇÃO DAS AMOSTRAS}

A mandioca (variedade IAC - 59/210, branca) foi colhida em plantações da Escola Superior da Agricultura Luiz de Queiroz (ESALQ), lavada, descascada, cortada em pedaços de $10 \mathrm{~cm}$ de comprimento e acondicionada em sacos de polietileno com $0,04 \mathrm{~mm}$ de espessura, num total de 24 amostras, com $550 \mathrm{~g}$ cada.

\subsection{IRRADIAÇÃO}

A irradiação das amostras foi efetuada no Centro de Energia Nuclear na Agricultura - USP (CENA), em fonte de Cobalto-60, tipo GAMMABEAM650 , atividade $1700 \mathrm{Ci}$, com taxa de dose $1050 \mathrm{~Gy} / \mathrm{h}$, por tempo suficiente para atingir as doses de 2; 4; 6; 8 e 10 kGy. 
Comparou-se as amostras irradiadas com uma amostra não irradiada (controle), preparada e mantida nas mesmas condições das anteriores a temperatura ambiente. Tais amostras foram acompanhadas durante 9 dias após a irradiação através da verificação da cor, aparência geral e sabor. Em cada avaliação também utilizou-se uma amostra denominada controle fresco, preparada nas mesmas condições das demais.

\subsubsection{Avaliação da Cor}

Determinada em colorímetro MINOLTA CHROMA METER - CR 200b (MINOLTA, RANSEY, N.J.), expressa em valor L de 0 (preto) a 100 (branco), no segundo dia após a irradiação.

\subsubsection{Avaliação Sensorial}

a) A aparência geral foi verificada por 5 provadores, pelo método de ordenação (7). Os provadores foram orientados para atribuirem notas de 1 a 5 , com valores: $1=$ péssima, $2=$ ruim, $3=$ regular, $4=$ boa, $5=$ excelente. As avaliações foram realizadas no $1^{\circ}, 2^{\circ}$ e $9^{\circ}$ dia após a colheita e irradiação.

b) No teste de aceitabilidade as amostras de mandioca cozidas por 30 minutos em um litro de água e $15 \mathrm{~g}$ de sal foram avaliadas por 20 provadores, pelo método da escala estruturada de 9 pontos (9), no qual atribui-se ao escore máximo a nota 9 ("extremamente aceitável") e ao mínimo a nota 1 ("extremamente inaceitável"). Submeteu-se os dados obtidos a análise de variância univariada e as variâncias foram discriminadas pelo teste $F(P<0,05)$. As comparações múltiplas entre as médias foram efetuadas pelos testes de Tukey $(7,8)$ e Dunnett (13).

\section{RESULTADOS E DISCUSSÃO}

\subsection{COR E APARÊNCIA GERAL}

Observa-se na Figura 1 que, no segundo dia, o controle apresentou valor $\mathrm{L}$ mais baixo em relação às amostras tratadas com irradiação, indicando escurecimento nas amostras não irradiadas. Durante a análise sensorial os provadores observaram manchas e pontos escuros nas amostras não irradiadas. As amostras irradiadas não apresentaram tais alterações, exceto leve tonalidade amarelada que decresceu em função do aumento da dose aplicada. A amostra irradiada com 10 kGy apresentou cor idêntica ao controle fresco (Figura 1). 
Quanto a aparência geral o controle obteve médias mais baixas, sendo consideradas ruins no segundo dia e péssimas aos 9 dias de armazenamento (Tabela 1). Além do escurecimento, foi observado avançado processo fermentativo. As amostras irradiadas não diferiram muito do controle fresco no segundo dia de armazenamento. Houve ligeiro decréscimo nas notas para as amostras que receberam doses de 2 a 6 kGy. Entretanto, após 9 dias, estas apresentaram manchas e pontos escuros, diferindo significativamente do controle fresco (Tabela 1). Por outro lado, as amostras irradiadas com 8 e 10 kGy mantiveram ótima aparência, mesmo no nono dia, não apresentando nenhum sinal de escurecimento.

A ausência de escurecimento na mandioca irradiada no segundo dia, em baixas doses e o posterior escurecimento nos dias seguintes, indicam que a atividade enzimática foi apenas retardada. Resultados semelhantes foram observados por BLUSHAN \& THOMAS (2), BOISEAU (3) e THOMAS (16). Observou-se também que, mesmo as amostras irradiadas com a menor dose não apresentaram problemas de fermentação durante o armazenamento. Tal fato justifica-se pela irradiação apresentar considerável ação germicida $(4,5)$.

\section{FIGURA 1 - MÉDIA DOS VALORES REFERENTES A COR DAS AMOSTRAS NO SEGUNDO DIA APÓS A IRRADIAÇÃO, MANTIDAS A TEMPERATURA AMBIENTE}

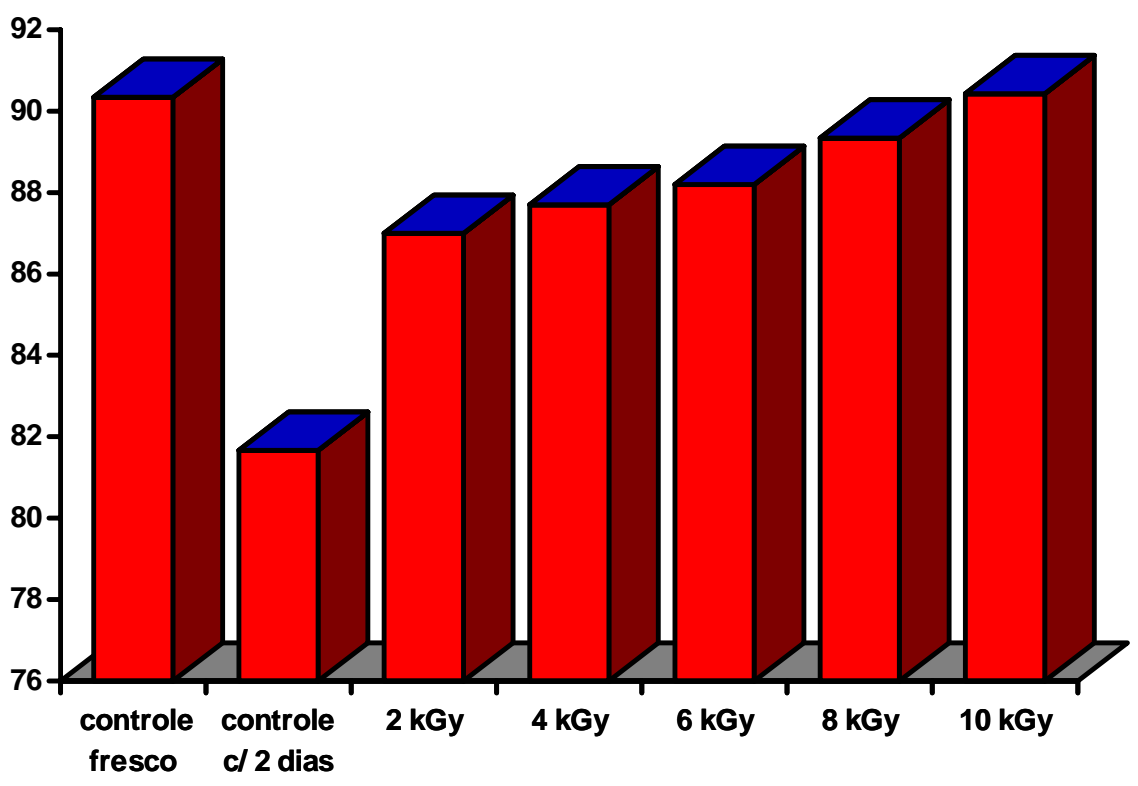


As amostras de mandioca foram provadas em três fases, ou seja, no primeiro dia (dia da colheita) avaliou-se somente o controle. No segundo dia avaliou-se todas as amostras irradiadas, repetindo-se a avaliação do controle que, embora já apresentasse sinais de escurecimento, ainda estava consumível. Finalmente, no nono dia, avaliou-se somente as amostras irradiadas com 8 kGy (Figura 2).

TABELA 1 - MÉDIAS DAS NOTAS ATRIBUÍDAS À APARÊNCIA GERAL DO CONTROLE FRESCO E DAS AMOSTRAS IRRADIADAS, NO SEGUNDO E NONO DIA, MANTIDAS A TEMPERATURA AMBIENTE

\begin{tabular}{c|c|c|c}
\hline Dias & $1^{\circ}$ & $2^{\circ}$ & $9^{\circ}$ \\
\hline 0 (controle) & $\mathbf{4 , 6 0}$ & 1,60 & 1,00 \\
\hline 2 & - & 3,80 & 1,70 \\
\hline 4 & - & 4,00 & 2,27 \\
\hline 6 & - & 4,10 & 2,47 \\
\hline 8 & - & $\mathbf{4 , 2 0}$ & $\mathbf{3 , 6 0}$ \\
\hline 10 & - & $\mathbf{4 , 8 0}$ & $\mathbf{4 , 0 4}$ \\
\hline
\end{tabular}

As notas atribuídas às amostras pelos provadores demonstraram que 0 controle, no segundo dia, apresentou resultados bem inferiores aos do controle fresco, confirmando a depreciação comercial do produto, ilustrada na Figura 1 e na Tabela 1. Já as amostras irradiadas com 2, 4 e 6 kGy apresentaram médias muito próximas ao controle fresco, indicando que estas doses não alteraram suas características organolépticas. Por outro lado, as amostras irradiadas com 8 e 10 kGy apresentaram resultados inferiores, em função de alterações na textura. A mandioca depois de cozida ficou mais dura, entretanto manteve a mesma aceitabilidade, inclusive no nono dia após a irradiação (Figura 2), ao contrário das demais amostras que nesse período já não apresentavam condições de consumo. Essa depreciação corrobora os resultados de THOMAS (16) obtidos em batatas irradiadas com baixas doses. Este verificou que a atividade enzimática depois de 10 dias volta a ser idêntica a do controle. A alteração na textura das amostras irradiadas com 8 e $10 \mathrm{kGy}$ foi ocasionada pela irradiação, a qual nestas doses pode modificar a cor, a textura, o sabor e o odor de certos alimentos $(12,14)$. Entretanto, segundo NEWSOME (9) a 
radiação não apresenta efeito significativo na digestibilidade de carboidratos.

FIGURA 2 - MÉDIAS DAS NOTAS DOS JULGADORES ATRIBUÍDAS À MANDIOCA COZIDA

\begin{tabular}{|llllllll|}
\hline 7.90 & 6.10 & 7.43 & 7.78 & 7.66 & 6.20 & 6.60 & 6.75 \\
\hline
\end{tabular}

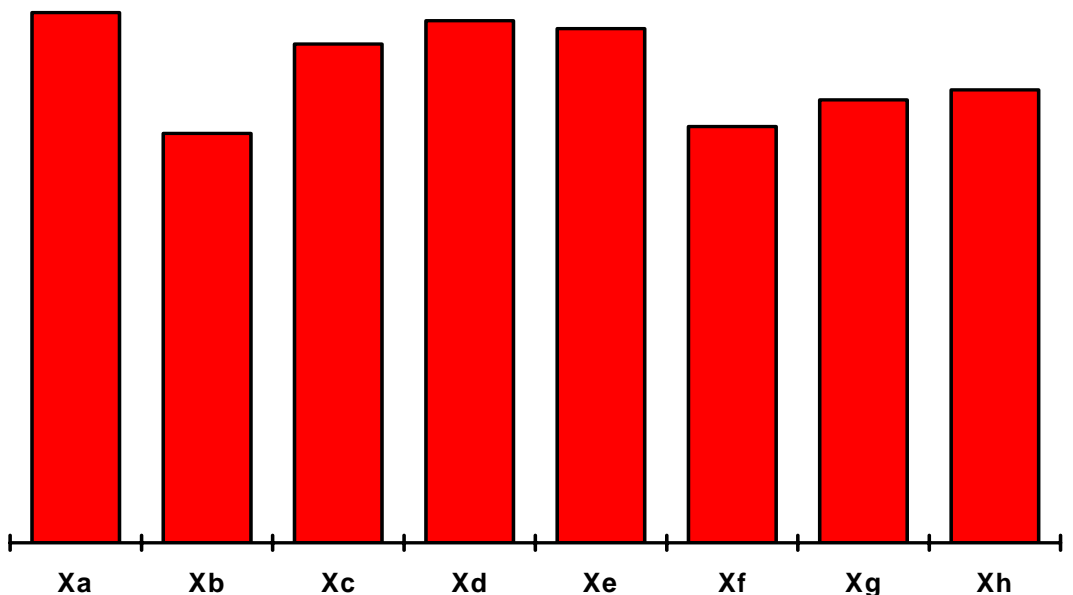

$\mathrm{Xa}=$ Controle fresco.

$\mathrm{Xb}=$ Controle com 2 dias armazenamento a temperatura ambiente.

$\mathrm{Xc}, \mathrm{Xd}, \mathrm{Xe}, \mathrm{Xf}$ e Xg = Amostras irradiadas com 2; 4; 6; 8 e $10 \mathrm{kGy}$ no segundo dia após a irradiação. $\mathrm{Xh}=$ Amostra irradiada com 8 kGy no nono dia após a irradiação.

TABELA 2 - VALORES DOS QUADRADOS MÉDIOS E SIGNIFICÂNCIA DO TESTE F PARA AS CARACTERÍSTICAS SENSORIAIS DA MANDIOCA IRRADIADA E DO CONTROLE

\begin{tabular}{lccccc}
\hline \multicolumn{5}{c}{ QUADRADOS MÉDIOS } \\
\hline $\begin{array}{l}\text { Causas da } \\
\text { variação }\end{array}$ & GL & SQ & QM & F (calc.) & F (tab.5\%) \\
\hline Amostras & 7 & 73,71 & 10,53 & $3,87 *$ & 2,01 \\
Provadores & 19 & 82,98 & 4,37 & 1,61 n.s. & \\
Resíduo & 133 & 361,93 & 2,72 & & \\
Total & 159 & 518,62 & & & \\
& & & & & \\
\hline
\end{tabular}

$\mathrm{GL}=$ Grau de liberdade. $\quad \mathrm{SQ}=$ Soma dos quadrados. $\quad \mathrm{QM}=$ Quadrado médio. $\mathrm{F}$ calc. $=\mathrm{F}$ calculado. $\quad \mathrm{F}$ tab. $=\mathrm{F}$ tabelado. $\quad$ ** $=$ Significância ao nível de $5 \%$. n.s. = não significativo

A análise estatística dos resultados revelou que houve diferença ao nível de $5 \%$ de significância, confirmada pelo teste de Tukey $(7,8)$ e pelo 
método de Dunnette, citado em STEEL and TORRIE (13), quando relacionou-se todas as amostras com o controle fresco (Tabela 2). Verificou-se diferença significativa entre o controle fresco e o controle com dois dias de armazenamento, assim como entre o controle e as amostras irradiadas com 8 kGy. Esta diferença, como já foi mencionada, justifica-se pela depreciação do controle, no segundo dia, mantido a temperatura ambiente e pela modificação de textura da amostra irradiada com 8 kGy. Entretanto, esta foi tão pequena que, aos nove dias de armazenamento já não apresentou significância (Tabela 3).

\section{TABELA 3 - DIFERENÇA DE VALORES MÉDIOS ENTRE AS CARACTERÍSTICAS SENSORIAIS DO CONTROLE E DEMAIS AMOSTRAS}

\begin{tabular}{ccc}
\hline & DIFERENÇAS & \\
\hline Xa - Xb & $1,80^{*}$ & $\alpha \beta$ \\
Xa - Xc & 0,47 & $\alpha$ \\
Xa - Xd & 0,12 & $\alpha$ \\
Xa - Xe & 0,24 & $\alpha$ \\
Xa - Xf & $1,70^{*}$ & $\alpha \beta$ \\
Xa - Xg & $1,30^{*}$ & $\alpha \beta$ \\
Xa - Xh & 1,15 & $\alpha$ \\
\hline $5 \%$ & 1,22 & \\
\hline
\end{tabular}

$\mathrm{Xa}=$ Contrle fresco.

$\mathrm{Xb}=$ Controle com 2 dias armazenamento a temperatura ambiente.

$\mathrm{Xc}, \mathrm{Xd}, \mathrm{Xe}, \mathrm{Xf}$ e $\mathrm{Xg}=$ Amostras irradiadas com 2; 4; 6; 8 e $10 \mathrm{kGy}$, no segundo dia após a irradiação.

$\mathrm{Xh}=$ Amostra irradiada com 8 kGy no nono dia após a irradiação.

* Teste de Dunnett ao nível de $5 \%$ de significância.

Os valores seguidos da mesma letra não diferiram estatisticamente do controle. Neste caso, as amostras consideradas diferentes apresentam entre as médias diferença superior a 1,22.

\section{CONCLUSÃO}

Através dos resultados obtidos pôde-se concluir que a irradiação com doses de 8 a 10 kGy inibe o escurecimento da mandioca, mantendo suas características físicas e sensoriais durante nove dias de armazenamento. 


\begin{abstract}
Cassava root was treated with gamma radiation at doses of 2, 4, 6, 8 and $10 \mathrm{kGy}$ to avoid enzimatic browning. The irradiated samples were kept for 9 days at room temperature and evaluated for color and sensorial analysis. Two days after harvest, the control sample showed black spots and alteration of organoleptic characteristics. The irradiated sample with 2 to 6 kGy showed good appearance and acceptability. However, after 9 days of storage, the control and the irradiated (2 to $6 \mathrm{kGy}$ ) samples were not safe to eat, only the irradiated cassava with doses of 8 and $10 \mathrm{kGy}$ did not show enzimatic browning and kept the good sensorial characteristics.
\end{abstract}

\title{
REFERÊNCIAS BIBLIOGRÁFICAS
}

1 BALAGOPALAN, C., PADMAJA, G.,NANDA, S. K., MOORTHY, S. N. Cassava in food, feed, and industry. Florida : CRC Press, 1988. $205 \mathrm{p}$.

2 BLUSHAN, B. and THOMAS, P. Effects of $\gamma$ irradiation and storage temperature on lipoxygenase activity and carotenoid disappearance in potato tubers (Solanum tuberosum L.). J. Agric. Food Chem., v.38, p. 1586-1590, 1990.

3 BOISSEAU, P. Irradiation and the food industry in France. Food Technology, p.138-140, May 1984.

4 BOKHARY, H. A., HASSIB, A. M. and SULEIMAN, A.A.A. Gamma irradiation effects on carbohydrate composition, growth of microorganisms and ESR spectra of gum arabic (Acacia senegal L.). Journal of Food Protection, v. 46, n. 7, p. 585-588, 1983.

5 CEREDA, M. P. Esterilização de amido de mandioca (Manihot utilissima, Pohl). Ciên. Tecnol. Aliment., Campinas, v. 4, n. 2, p. 139-157, 1984.

6 ELIAS, P. S. New concepts for assessing the wholesomeness of irradiated foods. Food Technology, v. 43, n. 7, p. 81-83, July 1989.

7 MONTEIRO, C. L. B. Técnicas de avaliação sensorial. 2. ed. Curitiba : CEPPA, 1984. $101 \mathrm{p}$.

8 MORAES, M. A. C. Métodos para avaliação sensorial dos alimentos. 7. ed. Campinas : 1990. 93 p.

9 NEWSOME, R. L. Perspective on food irradiation. Food Technology, v. 41, n. 2, p. 100-101, February 1987. 
10 PSZCZOLA, D. E. Food irradiation: countering the tacties and claims of opponents. Food Technology, v. 44, n. 6, p. 92-97, June 1990.

11 RAFFI, J., AGNEL, J. P. L., THIERY, C. J. et. al. Study of $\gamma$-irradiated starch derived from different foodstuffs: a way for extrapolating wholesomeness data. J. Agric. Food Chem., v. 29, n. 6, p. 12271232, 1981.

12 SPOTO, M. H. F., DOMARCO, R. E., WALDER, J. M. M., et. al. Radiação gama na conservação do suco concentrado de laranja. II. Características sensoriais. B. SBCTA., Campinas, v. 27, n. 2, p. 96-104, jul./dez.1993.

13 STEEL, R. G. D. \& TORRIE, J. H. Principles and procedures of estatistics. New York: [s.n.],1960. 481 p.

14 TAUHATA, L., ALMEIDA, E.S. Radiações nucleares: usos e cuidados. Niterói : CNEN, 1984. v.3

15 TEIXEIRA, C. G. Aplicação da radiação na preservação de alimentos. B. Cent. Trop. Tec. Alim., Campinas, v.10, p. 55-76, 1967.

16 THOMAS, P. Radiation preservation of foods of plant origin. Part 1. Potatoes and other tuber crops. CRC Critical Review in Food Science and Nutrition, v. 19, Issue 4, 1984.

\section{AGRADECIMENTOS}
1) Ao técnico LUIZ ANSELMO LOPES - CENA.
2) Ao Prof. Dr. JULIO MARCOS MELGES WALDER - CENA.
3) Ao CNPq e ao CEFET/PR pelo apoio financeiro.
4) A todos que direta ou indiretamente participaram desse trabalho. 